\section{Carotid plaque surface irregularities increase risk of ischemic stroke}

The presence of carotid stenosis is a wellestablished risk factor for ischemic stroke. There is some evidence that plaque surface morphology is an additional indicator of stroke risk; however, data from large population-based studies are lacking.

To examine the association between plaque surface morphology and risk of ischemic stroke, researchers from the Neurological Institute at Columbia University, New York, NY, conducted an analysis in the multiethnic (51.4\% Hispanic, 24.4\% black, 21.8\% white, $2.4 \%$ other) prospective Northern Manhattan Study cohort. High-resolution B-mode ultrasonography was used to examine carotid plaque surface characteristics in 1,939 individuals (mean age 68.6 years) with no history of stroke. Overall, 1,091 (56.4\%) individuals exhibited carotid plaque, with an irregular plaque surface being documented in 107 $(5.5 \%)$ subjects. After a mean follow-up of 6.2 years, $69(3.6 \%)$ of the study population had been diagnosed with ischemic stroke. Individuals with plaque surface irregularities had an $8.5 \%$ unadjusted cumulative 5-year risk of ischemic stoke, compared with $3.0 \%$ for those with regular plaque and $1.3 \%$ for those with no plaque. Following adjustment for age, sex, ethnicity, traditional vascular risk factors, degree of stenosis and plaque thickness, the presence of irregular plaque increased the risk of ischemic stroke fourfold compared with no plaque. Irregular plaque was also predictive of a combined vascular outcome comprising ischemic stroke, myocardial infarction and vascular death. On the basis of these results, the authors conclude that plaque surface irregularity could be a useful marker of vascular risk in addition to conventional risk factors.

Original article Prabhakaran S et al. (2006) Carotid plaque surface irregularity predicts ischemic stroke: the Northern Manhattan Study. Stroke 37: 2696-2701

\section{Long-term follow-up of a risk- adapted protocol for children with medulloblastoma}

Severe treatment-related mortality is a feature of current therapies for medulloblastoma. In this study, Gajjar et al. have assessed the longterm survival effects of a treatment protocol comprising risk-adapted radiotherapy followed by high-dose chemotherapy. Survival rates for patients with average-risk medulloblastoma ( $\leq 1.5 \mathrm{~cm}^{2}$ residual tumor, no metastatic disease) were comparable to those achieved under the longer treatment protocols of other studies, but morbidity was potentially reduced. In patients with high-risk medulloblastoma $\left(>1.5 \mathrm{~cm}^{2}\right.$ residual tumor or metastatic disease localized to neuraxis), a condition conferring a poor prognosis, survival rates were found to be higher than in most other reports.

The study evaluated outcomes for 134 children with medulloblastoma who sequentially received tumor resection, risk-adjusted craniospinal radiotherapy (23.4 Gy for average-risk disease; 36.0-39.6Gy for high-risk disease), and 4 months of high-dose chemotherapy. Estimated 5-year event-free survival was $83 \%$ for patients with average risk and $70 \%$ for those with high risk $(P=0.046)$. Tumor histological subtype also predicted event-free survival after adjustment for clinical stage, with large-cell anaplastic tumors conferring a poor survival rate (57\%) compared with classic histology (84\%) or desmoplastic tumor $(77 \% ; P=0.04)$.

In this protocol, both radiotherapy and chemotherapy were well tolerated, and there were no treatment-related deaths. The authors suggest that the high observed survival rates might be associated with the initiation of radiotherapy soon (within 4 weeks) after resection, and with the use of short-term dose-intensive chemotherapy following completion of radiotherapy.

Original article Gajjar A et al. (2006) Risk-adapted craniospinal radiotherapy followed by high-dose chemotherapy and stemcell rescue in children with newly diagnosed medulloblastoma (St Jude Medulloblastoma-96): long-term results from a prospective, multicentre trial. Lancet Oncol 7: 813-820 